\section{Research Square}

Preprints are preliminary reports that have not undergone peer review.

They should not be considered conclusive, used to inform clinical practice, or referenced by the media as validated information.

\title{
Genetic Diversity and Population Structure of 93 Rice Cultivars (Lines) (Oryza Sativa Xian Group) in Qinba in China by 3 Types of Genetic Markers
}

\author{
Yu Zhang ( $\nabla$ yuzhang20160315@outlook.com ) \\ Yewen Wang \\ Hanzhong Agricultral Research Institute \\ Peijiang Li \\ Hanzhong Agricultural Research Institute \\ Yuexing Wang \\ Xinjiang Agricultural University \\ Shimao Zheng \\ Shannxi University of Technology \\ Qiaoqiao He \\ Shannxi University of Technology \\ Xixi Zhou \\ Shannxi University of Technology
}

Shaanxi University of Technology https://orcid.org/0000-0002-5379-6773

\section{Research}

Keywords: Indica rice, Phenotypic traits, SSRs, SNPs, Genetic diversity, Population structure

Posted Date: November 12th, 2021

DOI: https://doi.org/10.21203/rs.3.rs-1050153/v1

License: (c) (i) This work is licensed under a Creative Commons Attribution 4.0 International License. Read Full License 


\section{Abstract}

Background: The Qinba region is the transition region between Indica and Japonica varieties in China. It has a long history of Indica rice planting of more than 7000 years and is also a planting area for fine-quality Indica rice. The aims of this study are to explore different genetic markers applied to the analysis population structure, genetic diversity, selection and optimization of molecular markers of Indica rice, thus providing more information for the protection and utilization on germplasm resources of Indica rice.

Methods: 15 phenotypic traits, a core set of 48 SSR markers as well as SNPs data obtained by genotyping-by-sequencing (GBS, Nlalll and Msel digestion, referred to as SNPS -Nlall $_{\text {and }}$ SNPs -Msel, $_{\text {, }}$ respectively) for this panel of 93 samples using the Illumina HiSeq2000 sequencing platform, were employed to explore the genetic diversity and population structure of 93 samples.

Results: The average of coefficient of variation (CV) and diversity index $\left(H_{e}\right)$ were $29.72 \%$ and 1.83 ranging from $3.07 \%$ to $137.43 \%$, and from 1.45 to 2.03 , respectively. The correlation coefficient between 15 phenotypic traits ranged from 0.984 to -0.604 . The first four PCs accounted for $70.693 \%$ phenotypic variation based on phenotypic analysis. A total of 379 alleles were obtained using SSR markers, encompassing an average of 8.0 alleles per primer. Polymorphic bands (PPB) and polymorphism information content (PIC) was $88.65 \%$ and 0.77 , respectively. The Mantel test showed that the correlation between the genetic distance matrix based on SNPs -Nlall $_{\text {and SNPs }}$-Msel was the largest $\left(R^{2}=0.88\right)$, and that based on 15 phenotypic traits and SSR was the smallest $\left(R^{2}=0.09\right)$. The 93 samples could be clustered into two subgroups by 3 types of genetic markers. Molecular variance analysis revealed that the genetic variation was $2 \%$ among populations and $98 \%$ within populations (the $N_{\mathrm{m}}$ was 0.16 ), Tajima's $\mathrm{D}$ value was 1.66 , the $F_{\mathrm{ST}}$ between the two populations was 0.61 based on 72,824 SNPs.

Conclusions: The population genetic variation explained by SNPs was larger than that explained by SSRs. The gene flow of 93 samples used in this study was larger than that of naturally self-pollinated crops, which may be caused by long-term breeding selection of Indica rice in the Qinba region. The genetic structure of the 93 samples was simple and lacked rare alleles.

\section{Introduction}

The two major types of Oryza sativa L. are classified as 0 . sativa Xian group (also known as Indica) and O. sativa Geng group (also known as Japonica) (Wang, W. et al. 2018), in which Indica rice is distributed mainly in the southern Qinling Mountains in China. The Qinba area is the climate transition area between the northern and southern areas as well as the transition area from Indica rice to Japonica rice, which is also the most suitable planting area for Indica rice in China. Germplasm resources form the basis of all breeding work; the analysis of genetic diversity and genetic structure is beneficial to mining excellent breeding materials and improving breeding efficiency. Particularly, in-depth genetic dissection of Indica rice germplasm resources have not been conducted. The population genetic structure is the non-random distribution of genes or genotypes in space and time, including genetic variations within populations and genetic differentiation between populations. Population structure analysis is essential to explore the biological adaptability, population formation process, evolutionary mechanism, protection, and development of biological resources. At the same time, populations with identical or similar genetic backgrounds is most suitable for genome-wide association studies (GWAS), therefore, the study of population genetic structure plays an important role in the field of biology, in which the selection of genetic markers is the top strategic priority, ranging from earlier morphological markers to more recent different types of DNA molecular markers (Delphine et al. 2010; Loveless et al.1984; Shen et al.2004; Yu et al. 2018). The Indica rice genome has simple sequence repeats (SSRs) that span approximately 10-50 kb (Ni et al. 2002; Yu et al. 2002). In the last few decades, SSR molecular markers have become important tools in the field of biology, particularly in terms of population structure, genetic mapping, and other related fields, SSR markers have also become the designated markers of the International Fingerprint Mapping Center (He et al. 2019; Huang et al.; 2016 Yin et al. 2008). These are employed in judicial identification, identification of new varieties of plants, such as rice, rape, and corn (Ceng et al. 2016; Li et al. 2004; Li et al. 2010; Lin et al. 2016; Sui et al. 2014; Wang et al. 2019). SSR markers are also used in DNA fingerprinting for breed protection (Teng et al. 2009). However, SSR markers are scarce, show unbalanced distribution in the genome, have weak electrophoretic resolution, and are relatively time-consuming and labor-intensive to study, and thus it is difficult to construct high-density genetic maps. With the recent development of next-generation sequencing technology, most biological studies have rapidly improved, in particular, the use of single nucleotide polymorphisms (SNPs) based on genome-wide scans. With the release of extensive rice genome sequencing data, one SNP in every hundred base pairs or even every dozens of base pairs has been identified, indicating that there are numerous SNPs in the rice genome (Nasu et al. 2002; Shen et al. 2004). A small number of SNPs can be used to resolve many problems, so the sequencing technology was born based on simplified genome by restriction site-associated DNA (RAD) tags (Miller et al. 2007). The frontrunner among these technologies is genotyping-bysequencing (GBS), which has recently gained attention because it utilizes methylation-sensitive restriction endonucleases (type II enzyme), thereby avoiding repetitive regions of the genome (methylated regions). GBS technology can rapidly identify high-density polymorphisms, especially SNPs (Elshire et al. 2011) In this study, two type II enzymes (N/alll and Msel) were selected by simulated whole-genome enzyme digestion, which generated RAD tags for sequencing to

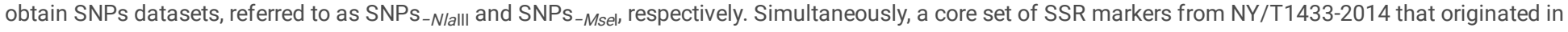
the Agricultural Standards of the People's Republic of China and 15 phenotypic traits were employed to explore gene flow and population genetic structure of 93 samples and to provide reference for future research studies using different genetic markers employed in related fields.

\section{Materials And Methods Plant Materials}

A total of 93 samples were collected from the Shaanxi Rice Research Institute (Hangzhong city, China), comprising 57 restoring lines, 19 maintainer lines, and 17 special rice, which were representative of the diversity of Oryza sativa Xian group present in the Qinba area in China. 


\section{Field Experiments}

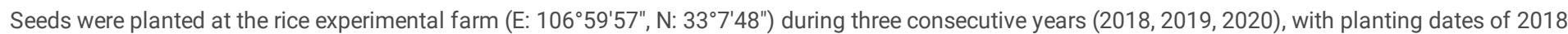
April 10, 2019 April 11, and 2020 April 8, and transplanted on May 24, May 24, May 20 according to a 16.7cm $\times 20 \mathrm{~cm}$ split-split-plot design. Each sample was arrayed randomly at plots with three repeats, to no edge row between the plots.

\section{Phenotyping}

Six plants in the middle of each plot were selected to investigate the values of agronomic, economic and quality traits according to "Recording items, methods and standards of national rice variety test and observation" as well as "National Standard of GBT 17891-1999 high quality paddy". The 15 selected phenotypic traits included sowing date, plant height, leaf length, leaf width, effective number of panicles per plant, panicle length, total number of grains per panicle, number of filled grains per panicle, 1000-grain weight, browning rate, milled rice rate, head milled rice rate, chalky grain rate, degree of chalkiness, and length/width ratio; the averages of the three-year data were used as the phenotypic data.

\section{Phenotypic traits statistical analysis}

The mean value ( $\nabla \mathrm{x})$, standard deviation $(\delta)$, and coefficient of variation $(\mathrm{CV})$ were computed. Shannon-Weiner index $(H)$ was calculated according to the following equation: $H^{\prime}=-\sum \mathrm{P}_{\mathrm{i}} \ln \mathrm{P}_{\mathrm{i}}$, where $\mathrm{P}_{\mathrm{i}}$ is the proportion of samples ranked at $\mathrm{i}^{\text {th }}$ grade for a given phenotypic trait among all samples (all of the phenotypic traits were divided into 10 grades by assigning values less than $\nabla x-2 \delta$ as 1 th grade and those greater than $\nabla x+2 \delta$ as 10 th grade, with inter-grade difference of $0.5 \delta$ for the remaining grades). All of phenotypic trait data were the standardized using Z-scores, and hierarchical cluster analysis was performed using between-groups linkage method based on Euclidean distance. The above analysis was carried out with the IBM SPSS statistics 22.0 software; MEGA7.0 software was used for editing and visualizing cluster results.

\section{SSR genotyping}

The genomic DNA of 93 samples was extracted from fresh leaves using the SDS technique and detected with $0.8 \%$ agarose gel electrophoresis. The 48 SSR primers were synthesized by Beijing Aoke Biotechnology Co., Ltd. (Beijing, China). PCR were carried out in a $10 \mu \mathrm{L}$ volume containing $1 \mu \mathrm{L}$ DNA template, $2 \mu \mathrm{L}$ $(10 \mu \mathrm{M})$ of forward and reverse primers $(1 \mu \mathrm{L}$ each), $5 \mu \mathrm{L} 2 \times$ Taq Master Mix, and $2 \mu \mathrm{L}$ RNase-free water. Reactions were programed as follows: initial denaturation at $94.0^{\circ} \mathrm{C}$ for 5 minutes, denaturation at $94.0^{\circ} \mathrm{C}$ for 1 minute, annealing at $50-60.0^{\circ} \mathrm{C}$ for 1 minute, and extension at $72.0^{\circ} \mathrm{C}$ for 1 minute, for a total of 35 cycles. Electrophoresis was performed using $8 \%$ non-denaturing polyacrylamide gel under $95 \mathrm{~V}$ voltage; bands were visualized via silver staining. Following electrophoresis, each amplification band corresponded to a primer hybridization locus and was considered as an effective molecular marker. Each polymorphic band detected by a same given primer represented an allelic mutation. In order to generate molecular data matrices, clear bands for each fragment were scored in every accession for each primer pair and recorded as 1 (presence of a fragment), 0 (absence of a fragment), and 9 (complete absence of band).

\section{SSR marker efficiency analysis}

The value of the polymorphism information content (PIC) was calculated using the PIC_Calc 0.6 program (http://www.bio-soft.net/dna/pic.htm). The level of polymorphism of each marker was assessed by the polymorphism information content, which measures the extent of genetic variation: PIC values smaller than 0.25 indicates low levels of polymorphism associated to a locus, PIC values between 0.25 and 0.5 imply moderate levels of polymorphism, while PIC values greater than 0.5 indicate high levels of polymorphism (Botstein et al. 1980).

\section{SNPs genotyping}

The genomic DNA of 93 samples was digested using the N/all and Msel enzymes. GBS was performed using the Illumina Hiseq 2000 platform of Novo Gene Bioinformatics Technology Co.,Ltd (Beijing, China). The SNPs data obtained with Nlalll and Msel digestion were recorded as SNPs Nlall, SNPs $_{-}$Msel, respectively. Polymorphism filtering of SNPs was done with dp., Miss and MAF of 2, 0.3 and 0.05 , respectively, followed by annotation based on the reference genome (ftp://ftp.ensemblgenomes.org/pub/plants/release-37/fasta/oryza_ indica/dna/).

Ld And Haplotype Construction

Genotype data were then used to calculate LD between SNPs and to construct haplotypes using the EM algorithm implemented in PLINK1.07 (https://www.cog-genomics.org/plink2). The commands "--r2" and "--blocks" were used to calculate LD and assign SNPs to their respective haplotypes by calculating inter-maker LD within a 200kb window, respectively. Figures were constructed using the Origin8 platform (http://www.originlab.com/).

\section{AMOVA and Gene flow}

A total of 72,824 SNPs were employed to analyze molecular variance (AMOVA) and gene flow. The components of variance attributable to different varieties and breeding lines were estimated from the genetic distance matrix using the Tajima \& Nei method, as specified in the AMOVA procedure in ARLEQUIN 3.1 (Excoffier et al. 2005). A nonparametric permutation procedure with 9999 permutations was used to test the significance of variance components associated with the different possible levels of genetic structure in this study. The pairwise Fst values, a value of F statistic analogs computed from AMOVA, were used to compare genetic distances between any two groups.

\section{PC clustering}

PC analysis was performed under the Eigen module using NTSYS-pc2.10e (Rohlf. 1998). 


\section{UPGMA clustering}

Identity-by-state (IBS) distance matrix generated by TASSEL5.0 (http://www.maizegenetics.net/tassel) was used to build an UPGMA tree. MEGA7.0 (http://http://www.megasoftware.net/) was used for editing and visualizing.

\section{Bayesian clustering}

STRUCTURE 2.3.4 (http://taylor0.biology.ucla.edu/structureHarvesteroybase.org/tools.php), which applies a Bayesian clustering algorithm, was used to simulate population genetic structure based on SSR and SNPs data, respectively. Using a membership probability threshold of 0.60 , population $\mathrm{K}$ values from 1 to 5 were simulated with 5 iterations for each K using 10,000 burn-in periods followed by 10,0000 Markov Chain Monte Carlo iterations in order to obtain an estimate of the most probable number of populations. Delta $\mathrm{K}$ was plotted against $\mathrm{K}$ values; the best number of clusters was determined following the method proposed by Evanno et al. (Evanno et al. 2005) and obtained via the Structure Harvester platform (http://taylor0.biology.ucla.edu/structureHarvester/) (Earl et al. 2012).

\section{Correlation analysis among genetic distance matrices by diffrent DNA marker dataset}

Mantel tests were used to measure the correlation between the genetic distance matrices generated using 15 phenotypic traits and SSR, 15 phenotypic traits

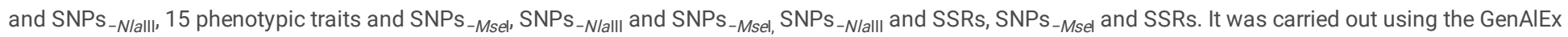
software with 9999 permutations (Peakall et al. 2012). $r \geq 0.9,0.8 \leq r<0.9,0.7 \leq r<0.8$, and $r<0.7$ represented significant correlation, moderate correlation, weak correlation, and no correlation, respectively.

\section{Results}

Phenotypic traits diversity and cluster analysis

Phenotypic diversity analysis

Data of the 15 phenotypic traits of the 93 samples are summarized in Table 1. The basic statistical analysis and diversity of the 15 phenotypic traits based on phenotypic data is shown in Table 2. The coefficient of variation (CV) was $29.72 \%$ on average and ranged from $3.07 \%$ (brown rice rate) to $137.43 \%$ (chalkiness). The average diversity index $\left(H_{e}\right)$ was 1.92 and ranged from 1.55 to 2.08 , with brown rice rate and chalkiness having lower $H_{e}$ indicating that there were relatively few phenotypes in these two traits. Overall, the Indica rice materials tested had relatively more phenotypes on these 15 traits; the distribution in each phenotype was uneven.

Table 1: Phenotypic data of 93 samples 


\begin{tabular}{|c|c|c|c|c|c|c|c|c|c|c|c|c|c|c|c|c|}
\hline Name & Type & 1 & 2 & 3 & 4 & 5 & 6 & 7 & 8 & 9 & 10 & 11 & 12 & 13 & 14 & 15 \\
\hline W1 & $\mathrm{R}$ & 115 & 128.2 & 46.26 & 2.58 & 6.17 & 28.95 & 241.36 & 217.64 & 28.57 & 79.14 & 67.47 & 58.60 & 17.50 & 4.00 & 2.40 \\
\hline W298 & $S$ & 115 & 128.2 & 46.26 & 2.58 & 6.17 & 28.95 & 241.36 & 217.64 & 28.57 & 79.14 & 67.47 & 58.60 & 17.50 & 4.00 & 2.40 \\
\hline W2 & $\mathrm{R}$ & 114 & 121.8 & 53.3 & 2.46 & 7.83 & 27.94 & 219.00 & 181.75 & 28.82 & 92.16 & 60.35 & 54.90 & 51.50 & 12.00 & 2.30 \\
\hline W300 & $S$ & 81 & 111 & 47.38 & 1.96 & 5.50 & 25.42 & 184.07 & 177.53 & 24.78 & 77.77 & 58.56 & 57.00 & 100.00 & 79.80 & 2.40 \\
\hline W352 & $S$ & 102 & 112.4 & 30.76 & 2.22 & 7.50 & 24.22 & 140.43 & 135.00 & 26.31 & 76.33 & 55.36 & 43.90 & 58.60 & 22.20 & 2.50 \\
\hline W353 & $S$ & 115 & 127.8 & 42.62 & 1.96 & 6.82 & 28.30 & 128.45 & 112.14 & 31.81 & 77.24 & 64.10 & 46.30 & 54.40 & 15.1 & 2.3 \\
\hline W354 & $S$ & 122 & 120.2 & 29.94 & 2.01 & 6.35 & 27.21 & 169.50 & 157.10 & 29.52 & 76.80 & 59.29 & 29.60 & 21.70 & 5.9 & 2.5 \\
\hline W355 & $S$ & 117 & 117.6 & 45.82 & 2.11 & 8.13 & 23.93 & 162.30 & 146.11 & 27.34 & 75.68 & 63.34 & 51.00 & 3.30 & 0.9 & 2.9 \\
\hline W357 & $S$ & 100 & 106 & 48.78 & 2.4 & 5.17 & 26.72 & 200.93 & 180.00 & 22.23 & 77.18 & 52.41 & 43.80 & 72.70 & 24.70 & 2.00 \\
\hline W359 & $S$ & 96 & 135.8 & 32.28 & 2.48 & 6.67 & 25.98 & 213.56 & 188.56 & 24.14 & 77.90 & 59.42 & 58.70 & 23.10 & 7.30 & 2.40 \\
\hline W361 & $S$ & 109 & 113.2 & 44.52 & 2.06 & 4.83 & 27.93 & 187.31 & 175.19 & 33.51 & 74.70 & 50.25 & 48.40 & 91.20 & 58.80 & 2.10 \\
\hline W366 & $S$ & 105 & 124.6 & 40.42 & 1.72 & 9.00 & 23.71 & 159.72 & 133.33 & 19.46 & 69.03 & 50.18 & 49.10 & 97.20 & 79.00 & 1.50 \\
\hline W367 & $S$ & 103 & 110 & 55.36 & 1.8 & 3.33 & 25.96 & 247.25 & 237.00 & 27.73 & 81.64 & 51.28 & 48.60 & 95.60 & 42.60 & 1.70 \\
\hline W369 & $S$ & 105 & 109.2 & 37.22 & 2.1 & 6.33 & 24.41 & 221.87 & 195.00 & 20.51 & 77.39 & 49.92 & 37.30 & 18.40 & 5.40 & 2.40 \\
\hline W370 & $S$ & 110 & 129.2 & 45.26 & 2.16 & 10.33 & 28.80 & 221.94 & 208.17 & 23.60 & 77.30 & 53.29 & 48.20 & 18.80 & 7.90 & 2.50 \\
\hline W375 & $S$ & 85 & 98.6 & 36.3 & 2.02 & 6.67 & 25.90 & 231.06 & 214.29 & 19.30 & 77.54 & 50.08 & 41.40 & 38.90 & 13.20 & 2.30 \\
\hline W377 & $S$ & 103 & 123 & 35.88 & 2.38 & 3.83 & 26.47 & 236.86 & 216.71 & 23.07 & 77.72 & 47.80 & 53.30 & 12.50 & 3.80 & 2.20 \\
\hline W380 & $S$ & 100 & 98 & 36.76 & 2 & 7.17 & 25.96 & 216.39 & 196.94 & 20.21 & 77.23 & 48.00 & 24.60 & 49.80 & 18.60 & 2.20 \\
\hline W381 & $S$ & 98 & 99.6 & 28.8 & 2 & 4.50 & 31.31 & 228.86 & 203.93 & 22.10 & 75.31 & 53.19 & 48.00 & 19.90 & 4.80 & 2.50 \\
\hline w3 & $\mathrm{R}$ & 116 & 133.2 & 45.34 & 2.38 & 6.33 & 29.28 & 216.14 & 201.36 & 36.22 & 79.87 & 51.44 & 28.90 & 37.60 & 9.30 & 2.40 \\
\hline W4 & $\mathrm{R}$ & 115 & 121.8 & 46.98 & 2.14 & 7.17 & 27.94 & 176.63 & 168.94 & 30.67 & 79.44 & 60.83 & 60.30 & 23.00 & 4.90 & 2.40 \\
\hline W5 & $\mathrm{R}$ & 113 & 125.6 & 46 & 2.28 & 6.00 & 31.35 & 254.55 & 247.36 & 26.83 & 78.58 & 56.36 & 46.70 & 17.70 & 4.10 & 2.50 \\
\hline W666 & $\mathrm{R}$ & 105 & 109.8 & 39.5 & 1.68 & 10.17 & 22.25 & 127.20 & 122.50 & 23.10 & 79.66 & 56.12 & 55.60 & 2.80 & 0.70 & 2.40 \\
\hline W667 & $\mathrm{R}$ & 101 & 105 & 35.6 & 2.43 & 6.83 & 22.04 & 175.00 & 168.50 & 24.41 & 78.62 & 60.95 & 55.90 & 25.70 & 7.70 & 2.10 \\
\hline W668 & $\mathrm{R}$ & 109 & 116.4 & 39.14 & 2.2 & 8.17 & 25.90 & 142.90 & 130.59 & 30.20 & 77.87 & 63.30 & 62.60 & 10.50 & 2.50 & 2.40 \\
\hline W669 & $\mathrm{R}$ & 109 & 110.8 & 37.56 & 1.78 & 10.33 & 24.84 & 140.95 & 115.29 & 21.67 & 78.60 & 64.80 & 64.10 & 4.00 & 0.80 & 2.70 \\
\hline W670 & $\mathrm{R}$ & 104 & 132 & 38.52 & 2.26 & 5.50 & 26.93 & 176.40 & 166.28 & 32.35 & 77.20 & 53.32 & 50.00 & 56.80 & 15.6 & 2.4 \\
\hline W671 & $\mathrm{R}$ & 110 & 128.6 & 45.76 & 2.62 & 6.17 & 28.33 & 190.31 & 176.38 & 31.60 & 80.06 & 60.73 & 59.40 & 53.70 & 15.60 & 2.30 \\
\hline W672 & $\mathrm{R}$ & 104 & 121.8 & 41.98 & 2.12 & 6.83 & 23.69 & 143.73 & 127.64 & 34.36 & 78.55 & 60.60 & 59.90 & 7.10 & 1.10 & 2.60 \\
\hline W673 & $\mathrm{R}$ & 106 & 119.6 & 37.83 & 2.15 & 8.00 & 26.10 & 128.07 & 120.21 & 30.95 & 72.24 & 55.13 & 52.70 & 7.80 & 2.10 & 2.40 \\
\hline W674 & $\mathrm{R}$ & 110 & 135.8 & 43.4 & 2.08 & 5.17 & 28.33 & 190.31 & 176.38 & 34.66 & 76.61 & 58.74 & 58.30 & 13.80 & 3.10 & 2.50 \\
\hline W675 & $\mathrm{R}$ & 110 & 118.6 & 30.84 & 1.98 & 8.00 & 27.53 & 154.08 & 141.33 & 29.10 & 78.89 & 57.35 & 55.20 & 22.80 & 5.00 & 2.40 \\
\hline W676 & $\mathrm{R}$ & 109 & 106 & 30.12 & 2.2 & 6.17 & 25.65 & 165.25 & 153.00 & 23.64 & 76.92 & 54.06 & 53.90 & 1.20 & 0.30 & 2.10 \\
\hline W677 & $\mathrm{R}$ & 110 & 112.2 & 30.12 & 2.1 & 8.17 & 24.23 & 174.44 & 159.63 & 29.13 & 79.80 & 58.10 & 56.60 & 18.10 & 3.50 & 2.30 \\
\hline W678 & $\mathrm{R}$ & 110 & 112 & 39.24 & 2.14 & 10.83 & 24.91 & 131.57 & 111.38 & 30.99 & 80.68 & 58.13 & 53.10 & 10.60 & 1.90 & 2.60 \\
\hline W679 & $\mathrm{R}$ & 112 & 108.8 & 38.18 & 2.3 & 7.83 & 24.72 & 133.44 & 120.94 & 29.62 & 78.82 & 60.50 & 59.60 & 19.90 & 4.50 & 2.60 \\
\hline W680 & $\mathrm{R}$ & 107 & 121.6 & 43.76 & 2.08 & 6.83 & 27.08 & 151.26 & 141.86 & 31.99 & 78.55 & 63.99 & 61.40 & 28.30 & 7.60 & 2.70 \\
\hline W681 & $\mathrm{R}$ & 111 & 116.8 & 37.56 & 2.3 & 5.17 & 24.72 & 130.73 & 119.00 & 30.08 & 76.89 & 54.86 & 54.00 & 1.60 & 0.20 & 2.30 \\
\hline W684 & $\mathrm{R}$ & 111 & 116.6 & 36.6 & 2.3 & 7.33 & 26.56 & 168.31 & 163.13 & 31.26 & 79.58 & 61.82 & 60.00 & 5.80 & 1.70 & 2.30 \\
\hline W685 & $\mathrm{R}$ & 110 & 121.4 & 31.94 & 2.44 & 8.17 & 23.98 & 176.87 & 169.53 & 33.26 & 79.48 & 56.74 & 54.50 & 35.20 & 10.00 & 2.10 \\
\hline W686 & $\mathrm{R}$ & 110 & 121 & 38.8 & 2.48 & 6.83 & 24.97 & 184.50 & 172.92 & 25.40 & 78.90 & 60.28 & 59.80 & 23.40 & 5.00 & 2.00 \\
\hline W687 & $\mathrm{R}$ & 110 & 108.4 & 38.22 & 2.12 & 7.33 & 25.07 & 142.33 & 128.87 & 28.74 & 78.56 & 56.34 & 54.10 & 29.50 & 6.90 & 2.20 \\
\hline W688 & $\mathrm{R}$ & 108 & 114.6 & 43.2 & 2 & 7.83 & 26.27 & 147.40 & 139.27 & 34.58 & 78.12 & 60.78 & 59.80 & 13.10 & 2.30 & 2.60 \\
\hline
\end{tabular}




\begin{tabular}{|c|c|c|c|c|c|c|c|c|c|c|c|c|c|c|c|c|}
\hline W689 & $\mathrm{R}$ & 109 & 105.2 & 36.54 & 2.54 & 8.67 & 25.75 & 131.06 & 86.86 & 30.03 & 80.33 & 54.00 & 39.80 & 29.40 & 5.80 & 2.20 \\
\hline W690 & $\mathrm{R}$ & 113 & 104.8 & 33.14 & 2.04 & 10.17 & 24.06 & 96.70 & 84.85 & 26.11 & 76.74 & 59.39 & 58.10 & 4.40 & 0.80 & 2.90 \\
\hline W691 & $\mathrm{R}$ & 108 & 115.6 & 44.72 & 2.02 & 8.83 & 28.18 & 135.40 & 130.30 & 34.40 & 78.35 & 56.20 & 51.50 & 9.40 & 1.90 & 2.60 \\
\hline W692 & $\mathrm{R}$ & 108 & 116.8 & 45.8 & 2.66 & 6.50 & 26.02 & 262.85 & 212.18 & 24.50 & 78.72 & 65.00 & 62.70 & 3.70 & 1.10 & 3.00 \\
\hline W693 & $\mathrm{R}$ & 114 & 122 & 39.16 & 2.52 & 7.17 & 24.98 & 234.75 & 208.13 & 27.27 & 77.99 & 58.61 & 57.20 & 23.90 & 8.90 & 2.20 \\
\hline W694 & $\mathrm{R}$ & 110 & 103.8 & 33.52 & 1.6 & 10.67 & 23.33 & 137.54 & 129.04 & 22.59 & 80.13 & 56.70 & 48.00 & 9.60 & 3.00 & 2.60 \\
\hline W697 & $\mathrm{R}$ & 105 & 90.8 & 27.32 & 1.82 & 7.33 & 22.96 & 114.19 & 101.31 & 21.27 & 79.24 & 62.57 & 61.80 & 2.60 & 0.70 & 2.40 \\
\hline W698 & $\mathrm{R}$ & 107 & 115.8 & 41.6 & 2.04 & 9.67 & 25.65 & 158.88 & 153.17 & 25.75 & 79.04 & 59.82 & 58.70 & 14.20 & 3.70 & 2.40 \\
\hline W699 & $\mathrm{R}$ & 102 & 108.8 & 80.7 & 2.22 & 3.83 & 26.27 & 428.50 & 394.60 & 20.63 & 78.94 & 61.29 & 58.80 & 9.50 & 2.40 & 2.60 \\
\hline W6 & $\mathrm{R}$ & 117 & 125.2 & 44.12 & 2.32 & 6.17 & 23.90 & 236.15 & 211.31 & 29.18 & 82.15 & 56.04 & 47.20 & 25.60 & 5.20 & 2.20 \\
\hline W700 & $\mathrm{R}$ & 101 & 121.8 & 35.6 & 2.72 & 7.17 & 24.09 & 166.38 & 153.81 & 25.17 & 79.40 & 63.40 & 62.00 & 1.60 & 0.30 & 2.60 \\
\hline W701 & $\mathrm{R}$ & 113 & 141.2 & 45.16 & 1.68 & 9.50 & 28.70 & 188.70 & 181.15 & 22.20 & 77.79 & 60.04 & 58.60 & 17.50 & 4.00 & 2.40 \\
\hline W702 & $\mathrm{R}$ & 107 & 126.6 & 33.08 & 1.78 & 10.83 & 21.27 & 127.13 & 118.13 & 23.38 & 78.85 & 63.27 & 61.40 & 0.70 & 0.20 & 2.60 \\
\hline W703 & $\mathrm{R}$ & 107 & 108.6 & 35.32 & 1.66 & 8.00 & 23.84 & 162.30 & 147.04 & 20.43 & 79.58 & 63.86 & 63.50 & 2.50 & 0.40 & 2.70 \\
\hline W704 & $\mathrm{R}$ & 114 & 133.4 & 45.7 & 1.9 & 5.40 & 24.22 & 206.46 & 196.77 & 25.36 & 78.24 & 56.96 & 56.20 & 5.80 & 1.10 & 2.40 \\
\hline W707 & $\mathrm{R}$ & 106 & 116.6 & 38.02 & 1.76 & 8.33 & 23.91 & 141.78 & 134.67 & 18.61 & 77.21 & 62.43 & 61.80 & 5.00 & 1.10 & 2.90 \\
\hline W708 & $\mathrm{R}$ & 104 & 117.4 & 33.16 & 2.26 & 8.17 & 26.06 & 235.25 & 216.00 & 23.70 & 76.51 & 61.23 & 59.10 & 7.41 & 1.23 & 2.60 \\
\hline W710 & $\mathrm{R}$ & 104 & 116.4 & 30.1 & 1.86 & 7.33 & 23.69 & 177.88 & 168.25 & 29.56 & 81.48 & 60.29 & 59.30 & 91.10 & 23.20 & 1.80 \\
\hline W711 & $\mathrm{R}$ & 102 & 115 & 32.88 & 1.82 & 8.17 & 22.98 & 159.88 & 153.24 & 28.85 & 81.24 & 67.08 & 65.80 & 89.10 & 21.20 & 1.80 \\
\hline W713 & $\mathrm{R}$ & 108 & 114.2 & 35.7 & 1.64 & 7.67 & 24.48 & 232.19 & 201.19 & 20.37 & 79.93 & 64.68 & 64.20 & 1.20 & 0.20 & 2.80 \\
\hline W714 & $\mathrm{R}$ & 100 & 120.2 & 26.94 & 1.9 & 7.33 & 21.90 & 200.82 & 181.59 & 19.69 & 79.96 & 59.60 & 57.70 & 3.00 & 0.70 & 2.40 \\
\hline W715 & $\mathrm{R}$ & 104 & 118.6 & 35.08 & 1.8 & 8.50 & 26.41 & 233.60 & 219.87 & 22.28 & 78.34 & 62.90 & 62.70 & 2.00 & 0.50 & 2.70 \\
\hline W716 & $\mathrm{R}$ & 92 & 104.4 & 39.22 & 2.08 & 6.67 & 24.20 & 175.50 & 145.70 & 28.09 & 78.25 & 62.24 & 56.80 & 13.70 & 3.40 & 2.70 \\
\hline W717 & $\mathrm{R}$ & 105 & 135 & 41.06 & 2.32 & 6.67 & 28.69 & 192.38 & 180.15 & 25.76 & 79.01 & 63.85 & 57.50 & 52.50 & 14.50 & 2.30 \\
\hline W718 & $\mathrm{R}$ & 113 & 141.4 & 36.28 & 2.1 & 10.17 & 26.83 & 153.96 & 137.42 & 27.43 & 79.26 & 61.09 & 59.90 & 2.20 & 0.30 & 2.70 \\
\hline W719 & $\mathrm{R}$ & 97 & 125.4 & 32.66 & 1.88 & 7.83 & 22.45 & 145.20 & 138.60 & 28.62 & 79.48 & 60.49 & 53.40 & 0.70 & 0.10 & 3.10 \\
\hline W720 & $\mathrm{R}$ & 99 & 122.4 & 32.56 & 2.04 & 9.17 & 24.35 & 112.14 & 104.83 & 29.21 & 77.50 & 58.73 & 54.70 & 2.00 & 0.30 & 2.90 \\
\hline W721 & $\mathrm{R}$ & 105 & 98.2 & 35.22 & 2.4 & 5.67 & 25.36 & 183.46 & 178.54 & 27.12 & 77.43 & 56.51 & 52.60 & 48.70 & 39.20 & 2.20 \\
\hline W722 & $\mathrm{R}$ & 96 & 123.6 & 37.06 & 2.18 & 6.17 & 26.69 & 159.75 & 110.56 & 24.65 & 79.20 & 55.98 & 15.30 & 39.50 & 10.30 & 2.90 \\
\hline W723 & $\mathrm{R}$ & 105 & 129.2 & 37.64 & 2.62 & 6.67 & 28.06 & 255.29 & 245.93 & 22.96 & 80.44 & 62.13 & 60.80 & 14.00 & 4.20 & 2.30 \\
\hline W724 & $M$ & 84 & 93.4 & 41.5 & 1.54 & 8.67 & 22.65 & 148.47 & 126.25 & 20.37 & 80.37 & 61.14 & 58.80 & 15.30 & 4.90 & 2.40 \\
\hline W725 & $M$ & 89 & 99.2 & 44.26 & 1.74 & 7.67 & 23.71 & 196.28 & 182.00 & 24.47 & 83.39 & 62.30 & 51.30 & 13.40 & 3.70 & 2.40 \\
\hline W726 & $M$ & 78 & 76.2 & 33.46 & 1.38 & 13.67 & 21.25 & 99.33 & 80.42 & 28.09 & 82.26 & 64.04 & 59.70 & 85.90 & 33.00 & 2.70 \\
\hline W727 & $M$ & 80 & 88.6 & 40.52 & 1.76 & 8.00 & 23.10 & 120.29 & 112.19 & 29.70 & 79.21 & 60.38 & 56.20 & 30.68 & 12.79 & 2.50 \\
\hline W728 & $M$ & 75 & 82.6 & 36.14 & 1.42 & 10.33 & 20.88 & 98.26 & 90.37 & 26.50 & 80.29 & 61.90 & 57.32 & 16.97 & 6.70 & 2.60 \\
\hline W730 & $M$ & 86 & 95.6 & 36.22 & 1.42 & 11.33 & 22.60 & 128.52 & 124.67 & 24.01 & 77.68 & 53.86 & 40.40 & 59.50 & 22.50 & 2.30 \\
\hline W732 & $M$ & 89 & 90.2 & 29.7 & 1.4 & 7.67 & 20.20 & 125.82 & 119.68 & 25.93 & 79.99 & 56.99 & 43.30 & 81.00 & 23.50 & 1.90 \\
\hline W733 & $M$ & 81 & 75.8 & 29.3 & 1.28 & 10.83 & 18.17 & 70.53 & 63.07 & 28.11 & 80.07 & 58.88 & 52.40 & 80.80 & 26.90 & 2.50 \\
\hline W734 & $M$ & 91 & 90.2 & 34.44 & 2.22 & 9.33 & 21.92 & 165.61 & 150.83 & 24.90 & 80.61 & 60.13 & 57.80 & 93.80 & 41.10 & 1.70 \\
\hline W735 & $M$ & 87 & 89.2 & 37.42 & 1.54 & 9.67 & 20.91 & 131.59 & 128.12 & 26.67 & 79.86 & 58.57 & 43.30 & 89.90 & 30.70 & 2.00 \\
\hline W736 & $M$ & 84 & 86 & 31.12 & 1.5 & 12.00 & 20.20 & 100.84 & 94.72 & 29.44 & 78.64 & 51.84 & 38.50 & 94.70 & 41.50 & 2.10 \\
\hline W737 & $M$ & 81 & 92 & 28.28 & 1.54 & 9.17 & 19.37 & 127.92 & 123.28 & 27.59 & 80.10 & 56.71 & 49.80 & 97.10 & 48.40 & 2.40 \\
\hline W738 & $M$ & 100 & 105.8 & 41.8 & 1.72 & 9.67 & 25.33 & 154.27 & 149.68 & 24.63 & 80.55 & 64.13 & 63.20 & 73.10 & 21.00 & 2.40 \\
\hline W739 & $M$ & 100 & 110.6 & 44.86 & 2.36 & 6.17 & 24.10 & 146.10 & 133.24 & 23.69 & 79.56 & 62.05 & 61.60 & 34.20 & 8.90 & 2.00 \\
\hline
\end{tabular}




\begin{tabular}{|llllllllllllllll|l|} 
W740 & M & 81 & 85.8 & 29.18 & 1.52 & 14.00 & 26.22 & 253.15 & 230.23 & 29.85 & 81.05 & 63.60 & 58.50 & 86.00 & 39.50 & 2.20 \\
\hline W741 & M & 85 & 86.6 & 34.18 & 1.54 & 10.83 & 19.78 & 82.93 & 71.67 & 30.37 & 76.67 & 57.31 & 52.30 & 93.40 & 45.20 & 2.20 \\
W742 & M & 104 & 132.8 & 36.88 & 2.44 & 7.50 & 27.73 & 262.47 & 253.33 & 22.38 & 79.80 & 62.17 & 61.20 & 12.30 & 3.10 & 2.40 \\
\hline W743 & M & 100 & 91.4 & 34.34 & 1.98 & 6.83 & 21.83 & 135.57 & 125.77 & 29.02 & 78.89 & 55.50 & 50.40 & 40.20 & 7.90 & 2.10 \\
\hline W744 & M & 95 & 92.8 & 50.86 & 1.74 & 7.00 & 24.79 & 133.40 & 127.07 & 31.90 & 77.38 & 57.72 & 52.90 & 27.60 & 5.50 & 2.10 \\
\hline W7 & R & 114 & 131.2 & 36.94 & 2.16 & 6.00 & 26.41 & 218.94 & 202.63 & 28.25 & 77.60 & 55.92 & 51.90 & 1.20 & 0.30 & 2.30 \\
\hline
\end{tabular}

Note: $\mathrm{M}, \mathrm{R}$ and $\mathrm{S}$ in the table refer to the maintainer line and restorer line of rice CMS Lines, and special rice. Phenotypic trait number (1 to 15) in first row correspond from left to the right to The period from seeding to heading (d); Plant heights $(\mathrm{cm})$; Leaf length ( $\mathrm{cm})$; Leaf width (cm); Average single plant valid spike number; Spike lengths (cm); Kernel numbers per spike; Grain numbe; 1000 -seed weights (g); Brown rice rate (100\%); Milled rice rate (100\%); Head rice rate (100\%); Chalky rice rate; Chalkiness; Length-width ratio.

Table 2: Basic statistical analysis and diversity of the 15 phenotypic traits

\begin{tabular}{|lllllll|}
\hline Phenotypic traits & Mean \pm SD & Median & Mode & Rang & $C V(\%)$ & $H_{e}$ \\
\hline The period from seeding to heading(d) & $102.95 \pm 10.543$ & 105 & 110 & 47 & 10.24 & 1.91 \\
\hline Plant heights $(\mathrm{cm})$ & $112.766 \pm 14.994$ & 115.600 & 121.8 & 65.6 & 13.30 & 2.05 \\
\hline Leaf length $(\mathrm{cm})$ & $38.6727 \pm 7.51025$ & 37.5600 & 30.12 & 53.76 & 19.42 & 1.89 \\
\hline Leaf width (cm) & $2.0423 \pm 0.33795$ & 2.0800 & 1.54 & 1.44 & 16.55 & 2.08 \\
\hline Average single plant valid spike number & $7.74 \pm 2.007$ & 7.50 & 6 & 11 & 25.83 & 2.03 \\
\hline Spike lengths (cm) & $25.07 \pm 2.593$ & 24.98 & 24 & 13 & 10.34 & 2.02 \\
\hline Kernel numbers per spike & $174.42 \pm 52.668$ & 165.61 & 190 & 358 & 30.20 & 1.92 \\
\hline Grain number & $159.68 \pm 49.365$ & 153.17 & 176 & 332 & 30.91 & 1.92 \\
\hline 1000-seed weights (g) & $26.75 \pm 4.129$ & 27.12 & 20 & 17 & 15.44 & 2.06 \\
\hline Brown rice rate (100\%) & $78.76 \pm 2.414$ & 78.85 & 79 & 23 & 3.07 & 1.79 \\
\hline Milled rice rate (100\%) & $58.80 \pm 4.439$ & 59.42 & 67 & 19 & 7.59 & 2.04 \\
\hline Head rice rate (100\%) & $53.61 \pm 8.955$ & 56.20 & 59 & 51 & 16.70 & 1.89 \\
\hline Chalky rice rate & $32.17 \pm 31.064$ & 19.90 & 1 & 99 & 96.56 & 1.68 \\
\hline Chalkiness & $11.97 \pm 16.45$ & 5 & 0 & 80 & 137.43 & 1.55 \\
\hline Length-width ratio & $2.39 \pm 0.293$ & 2.40 & 2 & 1 & 12.26 & 1.96 \\
\hline
\end{tabular}

Most phenotypic traits were correlated or significantly correlated. The most significant correlation was between kernel numbers per spike and grain number, followed by that between chalky rice rate and chalkiness. However, the correlation between chalky rice rate and length-width ratio was the least significant, followed by that between leaf width and average single plant valid spike number (Table 3 ).

Table 3: Pearson correlation coefficient analysis of the 15 phenotypic traits 


\begin{tabular}{|c|c|c|c|c|c|c|c|c|c|c|c|c|c|c|}
\hline Traits & 1 & 2 & 3 & 4 & 5 & 6 & 7 & 8 & 9 & 10 & 11 & 12 & 13 & 14 \\
\hline 1 & 1 & & & & & & & & & & & & & \\
\hline 2 & $0.726^{\star \star}$ & 1 & & s & & & & & & & & & & \\
\hline 3 & $0.224^{\star}$ & $0.249^{*}$ & 1 & & & & & & & & & & & \\
\hline 4 & 0.567 ** & $0.582^{\star \star}$ & $.261^{*}$ & 1 & & & & & & & & & & \\
\hline 5 & $-0.358^{* *}$ & $-0.398^{\star \star}$ & $-0.394^{\star *}$ & $-0.564^{* *}$ & 1 & & & & & & & & & \\
\hline 6 & $0.574^{\star \star}$ & $0.634^{* *}$ & $0.398^{\star *}$ & $0.571^{\star \star}$ & $-0.469^{\star *}$ & 1 & & & & & & & & \\
\hline 7 & $0.268^{\star \star}$ & $0.391^{\star \star}$ & $0.509^{\star \star}$ & $0.460^{\star \star}$ & $-0.520^{\star \star}$ & $0.554^{\star \star}$ & 1 & & & & & & & \\
\hline 8 & $0.260 *$ & $0.393^{\star \star}$ & $0.501^{\star *}$ & $0.428^{\star \star}$ & $-0.519^{\star \star}$ & $0.545^{\star \star}$ & $0.984^{\star \star}$ & 1 & & & & & & \\
\hline 9 & 0.179 & 0.111 & 0.074 & 0.159 & -0.024 & 0.147 & $-0.272^{\star \star}$ & $-0.250^{*}$ & 1 & & & & & \\
\hline 10 & -0.107 & -0.130 & 0.098 & -0.013 & 0.156 & -0.116 & 0.085 & 0.067 & 0.078 & 1 & & & & \\
\hline 11 & 0.053 & 0.095 & 0.049 & 0.014 & $0.222^{*}$ & -0.037 & -0.014 & -0.022 & 0.012 & $0.336^{\star \star}$ & 1 & & & \\
\hline 12 & 0.076 & 0.125 & 0.058 & 0.055 & 0.115 & -0.054 & 0.031 & 0.065 & -0.045 & 0.155 & $0.680^{\star \star}$ & 1 & & \\
\hline 13 & $-0.491^{\star \star}$ & $-0.414^{\star *}$ & -0.053 & $-0.322^{\star *}$ & 0.152 & $-0.268^{\star *}$ & -0.158 & -0.140 & 0.145 & 0.077 & $-0.239^{\star}$ & $-0.270^{\star *}$ & 1 & \\
\hline 14 & $-0.466^{\star \star}$ & $0.353^{\star \star}$ & -0.017 & $-0.301^{\star \star}$ & 0.116 & $-0.240^{*}$ & -0.116 & -0.099 & 0.036 & -0.143 & $-0.295^{\star \star}$ & $-0.208^{*}$ & $0.891^{\star \star}$ & 1 \\
\hline 15 & 0.095 & 0.171 & 0.005 & $0-.015$ & 0.156 & 0.104 & -0.047 & -0.084 & -0.042 & 0.001 & $0.360^{\star \star}$ & 0.134 & $-0.604^{\star *}$ & -0.51 \\
\hline
\end{tabular}

Note: Asterisk indicates significant difference between phenotypic traits using two-tailed t-tests. ${ }^{\star} P<0.05 ; * \star P<0.01$.

Principal components were extracted based on the criterion that the eigenvalue was greater than 1.0. The eigenvalues of the first four PCs in 15 phenotypic traits were greater than 1.0 , and together accounted for $70.693 \%$ of the phenotypic variation (Table 4). The first PC accounted for $31.527 \%$; the most important traits were spike lengths (0.167), plant heights (0.165) and leaf width (0.159). The second PC accounted for $18.137 \%$, the most important traits being lengthwidth ratio (0.252), milled rice rate $(0.244)$ and head rice rate $(0.195)$.

Table 4: Eigenvalue and contributive percentage of principal components and component scores coefficient matrix of the 15 phenotypic traits

\begin{tabular}{|c|c|c|c|c|}
\hline Traits code & $\begin{array}{l}\text { First principal } \\
\text { component }\end{array}$ & $\begin{array}{l}\text { Second principal } \\
\text { component }\end{array}$ & $\begin{array}{l}\text { Third principal } \\
\text { component }\end{array}$ & $\begin{array}{l}\text { Fourth principal } \\
\text { component }\end{array}$ \\
\hline The period from seeding to heading(d) & 0.155 & 0.052 & -0.212 & 0.135 \\
\hline Plant heights (cm) & 0.165 & 0.029 & -0.138 & 0.106 \\
\hline Leaf length (cm) & 0.108 & -0.098 & 0.188 & 0.130 \\
\hline Leaf width $(\mathrm{cm})$ & 0.159 & -0.038 & -0.091 & 0.156 \\
\hline Average single plant valid spike number & -0.136 & 0.155 & 0.050 & 0.020 \\
\hline Spike lengths (cm) & 0.167 & -0.066 & -0.083 & 0.081 \\
\hline Kernel numbers per spike & 0.155 & -0.136 & 0.262 & -0.151 \\
\hline Grain number & 0.152 & -0.141 & 0.264 & -0.137 \\
\hline 1000-seed weights (g) & 0.001 & 0.002 & -0.224 & 0.565 \\
\hline Brown rice rate (100\%) & -0.007 & 0.071 & 0.305 & 0.267 \\
\hline Milled rice rate $(100 \%)$ & 0.023 & 0.244 & 0.279 & 0.232 \\
\hline Head rice rate $(100 \%)$ & 0.030 & 0.195 & 0.272 & 0.194 \\
\hline Chalky rice rate & -0.121 & -0.242 & 0.099 & 0.244 \\
\hline Chalkiness & -0.110 & -0.248 & 0.077 & 0.142 \\
\hline Length-width ratio & 0.043 & 0.252 & -0.039 & -0.194 \\
\hline Eigenvalue & 4.729 & 2.721 & 1.763 & 1.391 \\
\hline 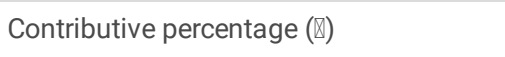 & 31.527 & 18.137 & 11.756 & 9.272 \\
\hline 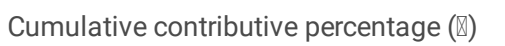 & 31.527 & 49.665 & 61.420 & 70.693 \\
\hline
\end{tabular}




\section{Phenotypic traits clustering}

Average Euclidean distance was 5.19, ranging from 0.90 (between W723 and W742) to 13.73 (between W699 and W733). Clustering result based on the 15 phenotypic traits was shown in Fig.1, which demonstrated that the 92 samples were clustered together in addition to W669 and showed a single genetic basis for the population.

\section{SSR marker analysis}

\section{Polymorphism of SSR markers}

A total of 378 bands was detected using 48 core SSRs primer pairs (Table 5). Among these, 336 polymorphic bands were detected. The average number of polymorphic fragments was 7, ranging from 1 to 14 . The highest number (14) of polymorphic bands was detected by RM278 while RM311 is the least bands. The average value of PPB (Percentage of polymorphic bands) was $88.87 \%$, ranging from $50 \%$ to $100 \%$. The average value of PIC (Polymorphism information content) was 0.77 , ranging from 0.19 to 0.88 . Data showed that core SSR in rice can produce rich bands and high polymorphic rate.

Table 5: Information and polymorphism of 48 SSR primers 


\begin{tabular}{|c|c|c|c|c|c|c|c|}
\hline Primer name & Chr. & Sequence $15^{\prime}-3^{\prime}[$ & Annealing temperature $\left({ }^{\circ} \mathrm{C}\right)$ & TNB & NPB & PPB (\%) & PIC \\
\hline RM583 & 1 & F:agatccatccctgtggagag; R:gcgaactcgcgttgtaatc & 55 & 10 & 10 & 100 & 0.86 \\
\hline RM71 & 2 & F:ctagaggcgaaaacgagatg; R:gggtgggcgaggtaataatg & 55 & 8 & 8 & 100 & 0.84 \\
\hline RM85 & 3 & F:ccaaagatgaaacctggattg; R:gcacaaggtgagcagtcc & 55 & 9 & 9 & 100 & 0.85 \\
\hline RM471 & 4 & F:acgcacaagcagatgatgag; R:gggagaagacgaatgtttgc & 55 & 8 & 6 & 75 & 0.86 \\
\hline RM274 & 5 & F:cctcgcttatgagagcttcg; R:cttctccatcactcccatgg & 55 & 12 & 12 & 100 & 0.84 \\
\hline RM190 & 6 & F:ctttgtctatctcaagacac; R:ttgcagatgttcttcctgatg & 55 & 5 & 5 & 100 & 0.74 \\
\hline RM336 & 7 & F:cttacagagaaacggcatcg; R:gctggtttgtttcaggttcg & 55 & 7 & 7 & 100 & 0.79 \\
\hline RM72 & 8 & F:ccggcgataaaacaatgag; R:gcatcggtcctaactaaggg & 55 & 12 & 9 & 75 & 0.86 \\
\hline RM219 & 9 & F:cgtcggatgatgtaaagcct; R:catatcggcattcgcctg & 55 & 2 & 2 & 100 & 0.36 \\
\hline RM311 & 10 & F:tggtagtataggtactaaacat; R:tcctatacacatacaaacatac & 55 & 2 & 1 & 50 & 0.37 \\
\hline RM209 & 11 & F:atatgagttgctgtcgtgcg; R:caacttgcatcctcccctcc & 55 & 4 & 3 & 75 & 0.67 \\
\hline RM19 & 12 & F:caaaaacagagcagatgac; R:ctcaagatggacgccaaga & 55 & 12 & 9 & 75 & 0.86 \\
\hline RM1195 & 1 & F:atggaccacaaacgaccttc; R:cgactcccttgttcttctgg & 55 & 8 & 8 & 100 & 0.84 \\
\hline RM208 & 2 & F:tctgcaagccttgtctgatg; R:taagtcgatcattgtgtggacc & 55 & 5 & 4 & 80 & 0.75 \\
\hline RM232 & 3 & F:ccggtatccttcgatattgc; R:ccgacttttcctcctgacg & 55 & 10 & 10 & 100 & 0.87 \\
\hline RM119 & 4 & F:catccccctgctgctgctgctg; R:cgccggatgtgtgggactagcg & 67 & 7 & 4 & 57.14 & 0.79 \\
\hline RM267 & 5 & F:tgcagacatagagaaggaagtg; R:agcaacagcacaacttgatg & 55 & 9 & 5 & 56.56 & 0.85 \\
\hline RM253 & 6 & F:tccttcaagagtgcaaaacc; R:gcattgtcatgtcgaagcc & 55 & 6 & 6 & 100 & 0.75 \\
\hline RM481 & 7 & F:tagctagccgattgaatggc; R:ctccacctcctatgttgttg & 55 & 7 & 7 & 100 & 0.80 \\
\hline RM339 & 8 & F:gtaatcgatgctgtgggaag; R:gagtcatgtgatagccgatatg & 55 & 8 & 8 & 100 & 0.79 \\
\hline RM278 & 9 & F:gtagtgagcctaacaataatc; R:tcaactcagcatctctgtcc & 55 & 14 & 14 & 100 & 0.85 \\
\hline RM258 & 10 & F:tgctgtatgtagctcgcacc; R:tggcctttaaagctgtcgc & 55 & 7 & 6 & 85.71 & 0.80 \\
\hline RM224 & 11 & F:atcgatcgatcttcacgagg; R:tgctataaaaggcattcggg & 55 & 8 & 8 & 100 & 0.84 \\
\hline RM17 & 12 & F:tgccctgttatttcttctctc; R:ggtgatcctttcccatttca & 55 & 9 & 9 & 100 & 0.78 \\
\hline RM493 & 1 & F:tagctccaacaggatcgacc; R:gtacgtaaacgcggaaggtg & 55 & 7 & 7 & 100 & 0.83 \\
\hline RM561 & 2 & F:gagctgtttggactacggc; R:gagtagctttctcccacccc & 55 & 8 & 5 & 62.50 & 0.85 \\
\hline RM8277 & 3 & F:agcacaagtaggtgcatttc; R:atttgcctgtgatgtaatagc & 55 & 7 & 7 & 100 & 0.75 \\
\hline RM551 & 4 & F:agcccagactagcatgattg; R:gaaggcgagaaggatcacag & 55 & 6 & 6 & 100 & 0.68 \\
\hline RM598 & 5 & F:gaatcgcacacgtgatgaac; R:atgcgactgatcggtactcc & 55 & 9 & 5 & 55.56 & 0.75 \\
\hline RM176 & 6 & F:cggctcccgctacgacgtctcc; R:agcgatgcgctggaagaggtgc & 67 & 10 & 7 & 70 & 0.88 \\
\hline RM432 & 7 & F:ttctgtctcacgctggattg; R:agctgcgtacgtgatgaatg & 55 & 5 & 5 & 100 & 0.71 \\
\hline RM331 & 8 & F:gaaccagaggacaaaaatgc; R:catcatacatttgcagccag & 55 & 8 & 7 & 87.50 & 0.82 \\
\hline OSR28 & 9 & F:agcagctatagcttagctgg; R:actgcacatgagcagagaca & 55 & 10 & 9 & 90 & 0.80 \\
\hline RM590 & 10 & F:catctccgctctccatgc; R:ggagttggggtcttgttcg & 55 & 9 & 6 & 66.67 & 0.87 \\
\hline RM21 & 11 & F:acagtattccgtaggcacgg; R:gctccatgagggtggtagag & 55 & 11 & 11 & 100 & 0.87 \\
\hline RM3331 & 12 & F:cctcctccatgagctaatgc; R:aggaggagcggatttctctc & 50 & 6 & 4 & 66.67 & 0.80 \\
\hline RM443 & 1 & F:gatggttttcatcggctacg; R:agtcccagaatgtcgtttcg & 55 & 10 & 7 & 70 & 0.75 \\
\hline RM490 & 1 & F:atctgcacactgcaaacacc; R:agcaagcagtgctttcagag & 55 & 9 & 9 & 100 & 0.82 \\
\hline RM424 & 2 & F:tttgtggctcaccagttgag; R:tggcgcattcatgtcatc & 55 & 5 & 5 & 100 & 0.72 \\
\hline RM423 & 2 & F:agcacccatgccttatgttg; R:ccttttcagtagccctccc & 55 & 7 & 7 & 100 & 0.82 \\
\hline RM571 & 3 & F:ggaggtgaaagcgaatcatg; R:cctgctgctctttcatcagc & 55 & 7 & 7 & 100 & 0.67 \\
\hline RM231 & 3 & F:ccagattatttcctgaggtc; R:cacttgcatagttctgcattg & 55 & 12 & 12 & 100 & 0.84 \\
\hline RM567 & 4 & F:atcagggaaatcctgaaggg; R:ggaaggagcaatcaccactg & 55 & 10 & 10 & 100 & 0.78 \\
\hline
\end{tabular}




\begin{tabular}{|c|c|c|c|c|c|c|c|}
\hline RM289 & 5 & F:ttccatggcacacaagcc; R:ctgtgcacgaacttccaaag & 55 & 10 & 10 & 100 & 0.88 \\
\hline RM316 & 9 & F:ctagttgggcatacgatggc; R:acgcttatatgttacgtcaac & 55 & 2 & 2 & 100 & 0.19 \\
\hline RM332 & 11 & F:gcgaaggcgaaggtgaag; R:catgagtgatctcactcaccc & 55 & 10 & 8 & 80 & 0.88 \\
\hline RM7102 & 12 & F:taggagtgtttagagtgcca; R:tcggtttgcttatacatcag & 55 & 3 & 3 & 100 & 0.43 \\
\hline
\end{tabular}

\section{Clustering based on SSR}

PC, in which the first three PC (eigenvalue) to select and their cumulative contribution of variance accounted for $15.76 \%$, and the unweighted pair-group method with arithmetic means (UPGMA) were performed, which demonstrated that the 93 genotypes could be divided into 2 subgroups (Fig. 2)

\section{Bayesian clustering based on SSR markers}

A total of 378 SSR bands was used to elucidate the population structure of the entire pool of 93 rice germplasms. The best $\mathrm{K}$ was $\mathrm{K}=2$, suggesting that the 93 rice germplasms were best divided into two subgroups (Fig. 3).

\section{SNPs marker analysis}

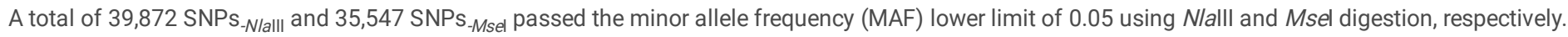

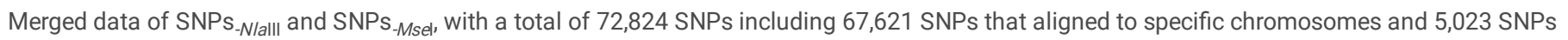
unlocalized, were then obtained.

\section{Linkage disequilibrium (LD) and haplotype analysis}

From the total of $6,288,753$ loci (93 samples $\times 67,621$ SNPs), 326,873 (5.198\%) were heterozygous. The 67,621 SNPs were unevenly distributed on the 12 chromosomes (Fig.4a); chromosome 1 contained the largest number of makers (8,425), while chromosome 8 included the least (3,953). LD, as represented by inter-loci $R^{2}$ values, was calculated for the 84,255 SNP pairs. $R^{2}$ value had a minimum of 0.2 and an average of 0.73 . 46,322 SNP pairs (54.98\%) had $R^{2}$ values higher than 0.8 , while 7,841 pairs $(9.31 \%)$ were in complete LD $\left(R^{2}=1\right)$. The 12 chromosomes yielded a total of 6,568 predicted haplotypes (Fig.4b), with chromosome 1 possessing the most haplotypes (776) and chromosome 10 possessing the least (349). The largest haplotype was composed of 95 SNPs. The longest haplotype spanned over $200.0 \mathrm{~kb}$; the average haplotype length was $33.71 \mathrm{~kb}$.

\section{AMOVA and gene flow}

The average MAF of the 93 samples was 0.21 . Tajima's D value was 1.66, which suggests low levels of both low- and high-frequency polymorphisms, indicating a decrease in population size and/or balancing selection that resulted in more haplotypes and lacked rare alleles in this population. Analysis of molecular variance (AMOVA) showed that the genetic variation was $98 \%$ within the population and $2 \%$ between populations, which indicated the existence of slight genetic variation among 93 samples. The genetic differentiation coefficient $\left(F_{\mathrm{ST}}\right)$ between the two populations was 0.61 , and gene flow $\left(N_{\mathrm{m}}\right)$ was 0.16 . Further investigation showed that the gene flow of selfing crops was the smallest, and that of annual herbaceous plants was the lowest. If $N_{\mathrm{m}}>1$, which indicates that the level of gene flow between populations is high, then genetic differentiation among populations is small; if $N_{\mathrm{m}}>4$, then gene communication between populations is more adequate and genetic differentiation is smaller; and $N_{\mathrm{m}}<1$ indicates that population differentiation may have occurred due to genetic drift. The gene flow was 0.16 , which indicates that the gene flow among populations in the Qinba region is lower, but nearly 2.5 -fold higher than that of conventional inbred plants, which may result in long-term artificial selection, leading to reduced genetic differentiation.

\section{Clustering based on SNPs}

\section{PC clustering}

Principal component analysis was performed to select the first three PCs (based on eigenvalue). Their cumulative contribution of variance accounted for

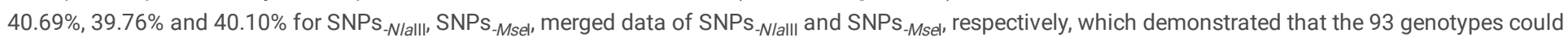
be clustered into two subgroups by the first three PCs (Fig.5), with W366 and W367 being always separated from other samples.

\section{UPGMA clustering}

The unweighted pair-group method with arithmetic means (UPGMA) algorithm was performed and demonstrated that the 93 genotypes could be divided into 2 subgroups (Fig. 6), which was consistent with the PC results. Group I included 1 to 3 samples, while group II contained 92 to 90 samples. The average genetic

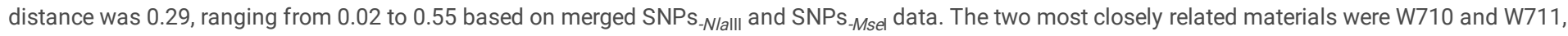
and the two most furthest materials were W366 and W740.

\section{Bayesian clustering}

72,824 SNPs (MAF $<5 \%$ ) were used to assess the population structure of the entire pool of 93 samples. Delta $\mathrm{K}$ reached a maximum value at $\mathrm{K}=2$, suggesting that the 93 samples were divided into two subgroups (consisting of 70 and 23 samples) (Fig. 7 ). In the population structure analysis, the results from $\mathrm{K}=2$ to 
$K=5$ revealed the occurrence of gene introgression between groupl and groupll, accounting for approximately $76.34 \%$ of the observed variations (calculated with $\mathrm{K}=2$ ).

\section{Clustering of different category materials}

Population genetic information of different category samples, including 57 restoring lines, 19 maintainer lines and 17 special rice lines was analyzed (Table 6 ) and clustered (Fig 8. 8a, 8b, 8c, respectively). Results showed that the genetic basis of the restorer line was more abundant than that of the maintainer line, and that the genetic basis of the special rice was wider than that of the conventional rice.

Table 6 Population genetic analysis of different category materials

\begin{tabular}{|llllll|}
\hline Samples & Tajima' & $\begin{array}{l}\text { Range of IBS genetic } \\
\text { distance }\end{array}$ & $\begin{array}{l}\text { The average genetic } \\
\text { distance }\end{array}$ & $\begin{array}{l}\text { Two samples with the closest } \\
\text { genetic distance }\end{array}$ & $\begin{array}{l}\text { Two samples with the farthest } \\
\text { genetic distance }\end{array}$ \\
\hline $\begin{array}{l}\text { Whole } \\
\text { materials (93) }\end{array}$ & 1.66 & $0.0229-0.5452$ & 0.3007 & W710/W711 & W366/W740 \\
\hline $\begin{array}{l}\text { Restoring lines } \\
(57)\end{array}$ & 1.36672 & $0.0229-0.3927$ & 0.2666 & W710/W711 & W685/W697 \\
$\begin{array}{l}\text { Maintainer } \\
\text { lines (19) }\end{array}$ & 0.43533 & $0.0242-0.3745$ & 0.2293 & W740/W741 & W375/W380 W738 \\
$\begin{array}{l}\text { Special rice } \\
(17)\end{array}$ & 0.62542 & $0.0285-0.5315$ & 0.3280 & W300/W366 \\
\hline
\end{tabular}

Correlation analysis of genetic distance matrices based on 3 types of genetic markers

All cluster analyses were based on the genetic distance or genetic similarity coefficient generated by genetic markers between samples; in the present study, the coefficients of correlation $\left(R^{2}\right)$ between the genetic distance matrices were $0.0914,0.1726,0.198,0.876,0.3478,0.2713$, respectively (Fig. 9). These results may be due to the use of different number of markers.

\section{Discussion}

Phenotype is the result of the interaction of genotype with environment. A given genotype can be expressed as different phenotypes in different environments; this is known as the plant inherent phenotypic plasticity, which is different from the genotype. In the study of germplasm resources with more samples, economical and effective method with the use of phenotype data to study population genetic structure and genetic diversity is also a very important at this early stage. The selected 15 traits belong to quantitative traits and are greatly affected by environment; hence it is not recommended to use them for population genetic structure analysis. In recent decades, SSR markers, which represent second-generation DNA molecular markers, have been widely used for plant population genetic analysis, phylogenetic reconstruction, and quantitative trait mapping. All kinds of DNA markers are different and results generated by different DNA markers reflect different polymorphic region in the genome and can reveal various information contained in the genome. Theoretically, the more markers used, the more accurate results will be. SSR markers are mostly distributed in centromeres, telomeres, introns, and 3' untranslated regions (UTR). Most of these markers are non-functional genetic markers and do not affect the application of SSR marker in clustering analysis. While the population genetic variation explained by SNPs was larger than that explained by SSRs (for example, in PC analysis), the accumulative contribution rate of the first three major factors analyzed by SSR was only $15.76 \%$, far less than that using SNPs data (40.10\%), indicating that the more DNA polymorphism, the more accurate the population variation can be explained. For association analysis between markers and traits, greater number of polymorphic sites is associated with higher mapping resolution. A natural population often contains multiple sub-populations, which could result in high degree of LD within the tested population and lead to pseudo-association between markers and traits. Therefore, LD and haplotype studies are necessary before carrying out association analysis. The core 48 pairs of SSR markers as well 72,824 SNPs had rich bands and high polymorphism in Indica rice genome; clustering result of SSR was concordant with that of SNPs, but different from phenotypic traits clustering.

All analyses of the population genetic structure were based on the estimation of genetic distance or genetic similarity coefficient matrix between samples. Analyses were conducted using three methods: PC, UPGMA, and Bayesian clustering. Bayesian algorithm is more practical than UPGMA and PC analysis no matter which genetic marker is used given the prior pedigree knowledge of the 93 samples. At the same time, the size of gene flow of each sample can be seen from the population genetic structure graph based on Bayesian algorithm.

Through the analysis of different types of materials (57 restorer lines, 19 sterile lines, and 17 special rice), the results showed that the genetic basis of the restorer lines was richer than that of the maintainer lines, which was consistent with the conclusion of Ying Jiezheng et al. (Ying et al. 2007). The main reason may be that most CMS (cytoplasmic male sterile) lines currently used in production are related to cultivars such as Zhenshan 97B, 区-32B, Zhong9a and Gang46a and may be derived from Aizazhan and Aijiaonante, which originated from dwarf rice varieties. At present, the restorer lines used in combination production originate from the Yangtze River Basin of China, Sichuan, Southeast Asia, South Korea, etc, and were created by crossing Indica and Japonica rice. Special rice has an abundant genetic basis compared to other rice germplasm resources and has high breeding potential.

\section{Conclusions}

Higher number of markers is related to higher explained population variation. Clustering results based on different genetic markers showed that the genetic basis of 93 samples was single. Average genetic distance was 0.29 based on 72,824 SNPs of 93 samples, which may be due to many reasons, such as the 
wide exchange of variety resources among breeding units in the process of breeding, and similar breeding goals. Genetic effects in populations depend on the opportunity distribution of MAFs across the genome-wide, and different populations have different MAF values. Although the gene flow in the population composed of 93 samples was relatively large, the average MAF of the population was only 0.21 , indicating the genetic structure of 93 samples is simple and lacked rare alleles. Measures to improve the genetic diversity of rice cultivars in the Qinba area are important.

\section{Abbreviations}

AMOVA: Analysis of molecular variance; DNA: Deoxyribonucleic acid; GWAS: genome-wide association studies; GBS: genotyping by sequencing; IBS: Identity by state; LD: Linkage disequilibrium; MAF: Minor allele frequency; NPB: number of polymorphic bands; PPB: Percentage of polymorphic bands; PIC: Polymorphism information content; PCA: Principal component analyses; RAD: restriction site-associated DNA; SNP: Single nucleotide polymorphism; SSR: Simple sequence repeats; TNB: total number of bands; UPGMA: Unweighted pair group method with arithmetic mean

\section{Declarations}

\section{Acknowledgments}

We thank LetPub (www.letpub.com) for its linguistic assistance during the preparation of this manuscript.

\section{Authors' contributions}

Yu Zhang wrote the manuscript, Yewen Wang and Peijiang Li performed the field experiments, Yuexing Wang, Shimao Zheng, Qiaoqiao He and Xixi Zhou performed the laboratory tests and analyzed data.

\section{Funding}

This study was supported by the Sci-technological Project of Shaanxi Province (NYKJ-2016-35), the Sci-technological Project of Shaanxi Province (2013K0210-01), the Sci-technological Project of Shaanxi Province (2020NY-050), the Sci-technological Project of Shaanxi Province (2019NY-041)

\section{Ethics approval and consent to participate}

All authors read and approved the manuscript.

\section{Consent for publication}

All authors agreed to publish this manuscript.

\section{Competing interests}

The authors declare that they have no competing interests.

\section{Author Details}

${ }^{1}$ Shaanxi University of Technology, 72300 Hanzhong Shaanxi, China; Shaanxi Province Key Laboratory of Bio-resources, 72300 Hanzhong Shaanxi, China; QinLing-Bashan Mountains Bioresources Comprehensive Development C. I. C., 72300 Hanzhong Shaanxi, China; Qinba State Key Laboratory of biological resources and ecological environment, 72300 Hanzhong Shaanxi, China.

${ }^{2}$ Hanzhong Agricultural Sciences Institute, 723000 Hanzhong Shaanxi,China.

${ }^{3}$ College of Agronomy, Xinjiang Agricultural University, 830052 Urumqi, China.

\section{References}

1. Agricultural industry standard of the people's Republic of China(NY/T1433-2014),Protocol for identification of rice varieties-SSR marker method

2. Botstein D, White RL, Skolnick M, Davis RW (1980) Construction of a genetic linkage map in man using restriction fragment length polymorphisms. Am J Hum Genet.32(3):314-331

3. Zeng X, D Peng, Shi Y, Xie W, Liu AM.(2016) Fingerprinting Construction of Rice Core Parental Lines with SSR Markers. Crop Research 30:481-486+511

4. Delphine VI, Albrecht EM, Claude L, Benjamin S. (2010) Population structure and genetic diversity in a commercial maize breeding program assessed with SSR and SNP markers. Theoretical and Applied Genetics 120: 1289-1299

5. Earl, Dent A. and vonHoldt, Bridgett M. (2012) STRUCTURE HARVESTER: a website and program for visualizing STRUCTURE output and implementing the Evanno method. Conservation Genetics Resources 4:359-361.

6. Elshire R.J., Glaubitz J.C., Sun Q., Poland J.A., Kawamoto K., Buckler E.S., and Mitchell S.E. (2011) A robust, simple genotyping-by-sequencing (GBS) approach for high diversity species. PLoS One 6(5)

7. Evanno G, Regnaut S, Goudet J. (2005) Detecting the number of clusters of individuals using the software STRUCTURE: a simulation study. Mol Ecol $14: 2611-2620$ 
8. Excoffier L, Laval G, Schneider S. (2005) Arlequin: an integrated software package for population enetics

9. data analysis. Version 3.0. Computational and Molecular Population Genetics Laboratory (CMPG).

10. Berne (Switzerland): Institute of Zoology, University of Berne

11. He GL, Fu GP, Peng CS, Deng W, Zhu S, Yang Y, Wei YQ,Chen LM, Shen LJ, Shen XH, Huang RL (2019) DNA Fingerprint Map and Analysis of Genetic Diversity of the Japonica Rice Varieties in the Regional Test in Jiangxi Province in 2018. Acta Agriculturae Universitatis Jiangxiensis 41:843-852

12. Huang X, Lizhi Li, Zhang J, Danlin, H. E., Zhang, X., Chen J. (2016) Evaluation of Diversity and Evolution of the Microsatellite LEI0258 in Chicken Region from South China. Chinese Journal of Animal \& Veterinary Sciences

13. Li C, Zhang Y, Ying K, Liang XL, Han B. (2004) Sequence variations of simple sequence repeats on chromosome 4 in two subspecies of the Asian cultivated rice. Thear Appl Genet. 108: 392-400

14. Li HB, Yang J, ZW, L. v., Bin Y I, Wen J, Fu T D, Tu J X, Ma C Z, Shen J X. (2010) Screening of Brassica napus core SSR primers. Chinese Journal of Oil Crop Sciences 32:329-336

15. Loveless MD, Hamrick JL. (1984) Ecological determinants of genetic structure in plant populations. Annu.Rev.Ecol.Syst. 15:65-95

16. Lin YX, Wang AX, Liu H, Wang Z, Liang MZ, Dai XJ, et al. (2016) Research on DNA Molecular Digital Fingerprint Database Based on 48 Pairs of SSR Primers for 94 Hybrid Rice Parents in NYT 1433-2014. Chinese Journal of Rice Science 30:593-602

17. Miller M R, Dunham J P, Amores A, WA Cresko, EA Johnson (2007) Rapid and cost-effective polymorphism identification and genotyping using restriction site associated DNA (RAD) markers. Genome Research 17: 240-248

18. Nasu S, Suzuki J, Ohta R, Hasegawa K, Yui R, Kitazawa N, Rika. (2002) Search for and analysis of single nucleotide polymorphisms (SNPS) in rice (Oryza sativa, Oryza rufipogon) and establishment of SNP markers. DNA Res. 9: 163-171

19. Ni J, Peter M Colowit, DJ Mackill. (2002) Evaluation of Genetic Diversity in Rice Subspecies Using Microsatellite Markers. Crop Science 42:601-607

20. Peakall R, Smouse PE. (2012) GenAlEx 6.5: genetic analysis in Excel. Population genetic software for teaching and research-an update. Bioinformatics 28:2537-2539

21. Rohlf F J. (1998) NTSYS-pc. Numerical Taxonomy and Multivariate Analysis System, Version 2.02. Exeter Software, New York

22. Shen YJ, Jiang H, Jin JP, Zhang ZB, Xi B, He YY. (2004) Development of genome-wide DNA Polymorphism database for map-based cloning of rice genes. Plant Physial 125:1198-1205

23. Sui GL, Yu SC, Yang JX, Wang WH, Su TB, Zhang FL, DS Zhang, XY Zhao. (2014) Validation of a Core Set of Microsatellite Markers and Its Application for Varieties Identification in Chinese Cabbage. Acta Horticulturae Sinica. 41:2021-2034

24. Teng HT, Lu B, Zhao JR, Xu Y, XY Li. (2009) DNA Fingerprint Profile Involved in Plant Variety Protection Practice. Biotechnology Bulletin 1:1-6

25. Wang MH, Zhang XT, Wu GL, Jiang Q, Shi YH. (2019) DNA Fingerprints Construction and Purity Identification Based on SSR Markers for Rice Varieties in Ningbo City. China Rice 25:50-54

26. Wang, W., Mauleon, R., Hu, Z. Hleung. (2018) Genomic variation in 3,010 diverse accessions of Asian cultivated rice. Nature 557:43-49

27. Yin Q Q, Da-Yu L I, Wang H Z, Cao D C, LU Cui-Yun, XW Sun, LQ Liang (2008) Microsatellite marker analysis on genetic diversity in two German mirror carp(Cyprinus carpio L.) families. Journal of Anhui Agricultural University

28. Ying JZ, Shi YF, Zhuang JY, Xue QZ. (2007) Microsatellite Marker Evaluation on Genetic Diversity of the Major Commercial Rice Varieties in China. Scientia Agricultura Sinica 4:649-654

29. Yu J, Hu S, Wang J, Wong G, Li SG, Liu B, Deng Y, Zhou Y, Zhang X (2002) A draft sequence of the rice genome (Oryza sativa L. ssp. indica). Science 296: 79-92

30. Yu Zhang, Xiaojuan Zhang, Xi Chen,Wang Sun, Jiao Li (2018) Genetic diversity and structure of tea plant in Qinba area in China by three types of molecular markers. Hereditas 155:22

\section{Figures}
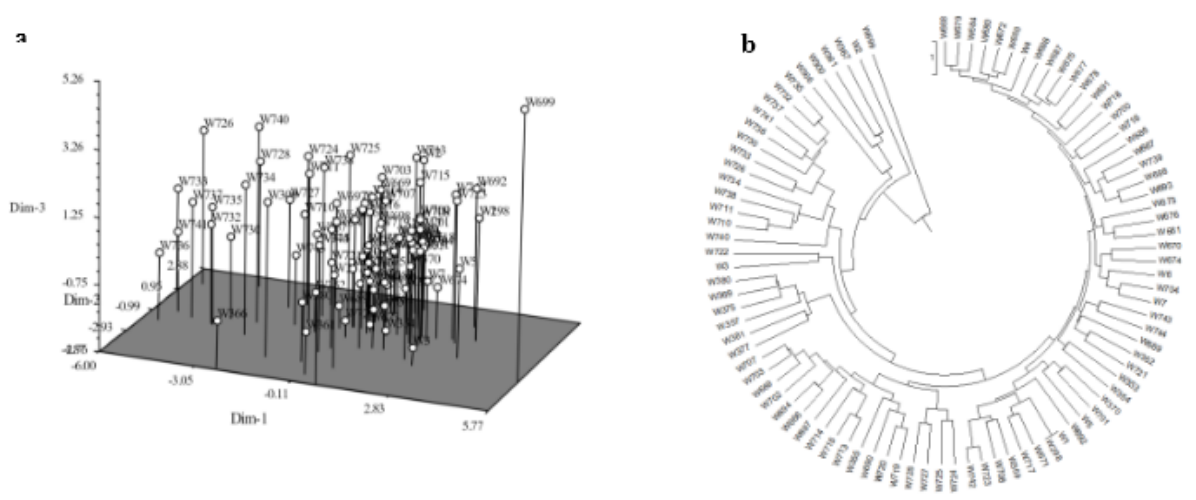

Figure 1

Page $14 / 17$ 
Cluster diagram based on the 15 phenotypic traits. a. via PC clustering; b. via UPGMA clustering Average Euclidean distance was 5.19 , ranging from 0.90 (between W723 and W742) to 13.73 (between W699 and W733). Clustering result based on the 15 phenotypic traits was shown in Fig.1, which demonstrated that the 92 samples were clustered together in addition to W669 and showed a single genetic basis for the population.

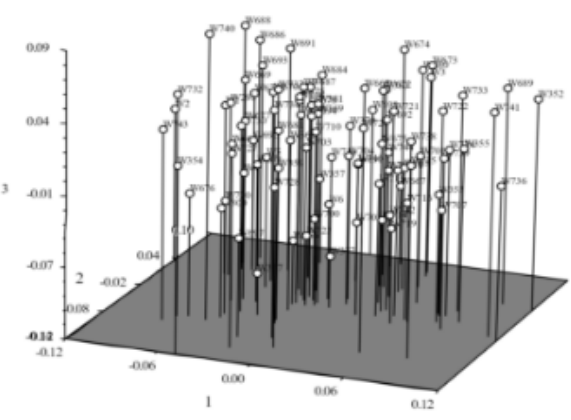

b

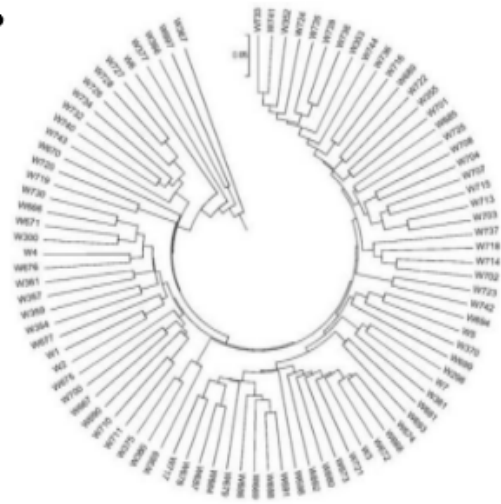

Figure 2

The cluster diagram based on SSR. a. by the PC clustering; $b$. by the UPGMA clustering
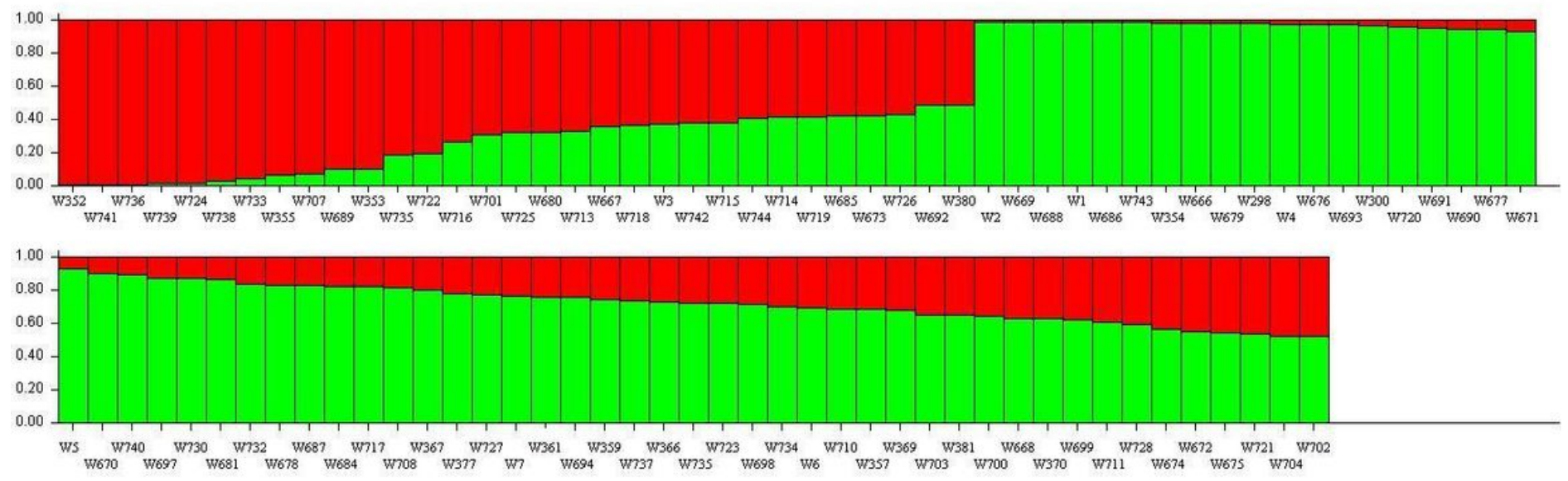

Figure 3

Bayesian clustering based on SSR markers; Red: group I; Green: group II. Each vertical line on the X-axis correspond to a sample. The proportion of each color represents probability rate with which a given genotype belongs to each group.

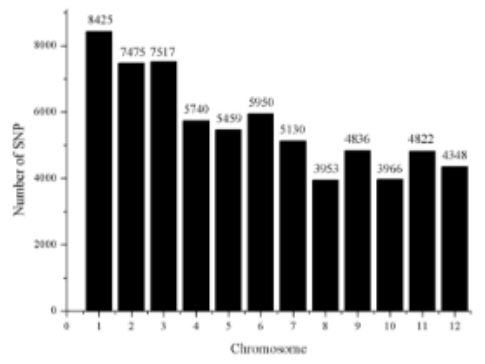

b

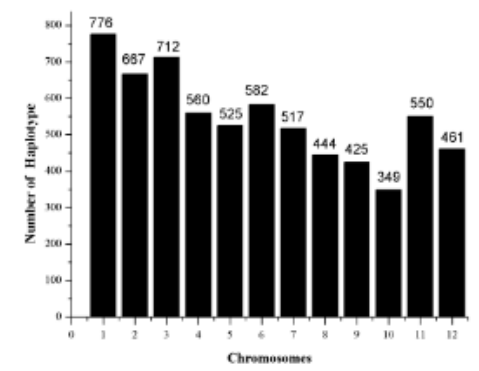

Figure 4

The distribution of SNPs and haplotype among the 12 chromosomes. a. SNPs distribution. b. Haplotype distribution. 
a

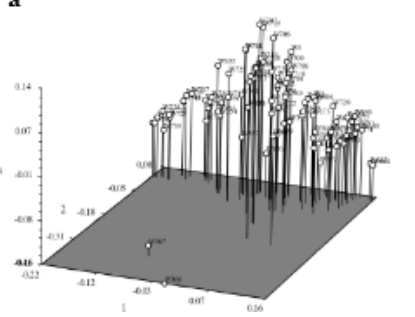

b

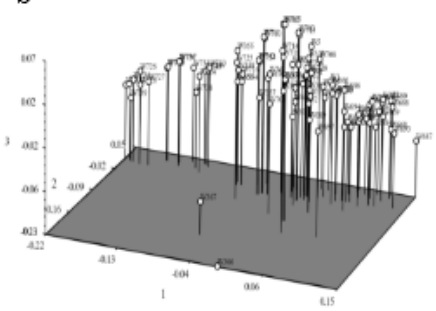

c

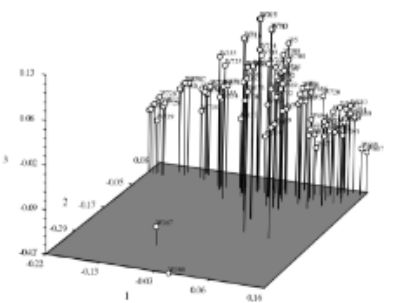

Figure 5

PCA plots using the different SNPs markers. a. by SNPs-Nlalli; b. by SNPs-Msel; c. by merged SNPs-Nlalll and SNPs-Msel data.

a

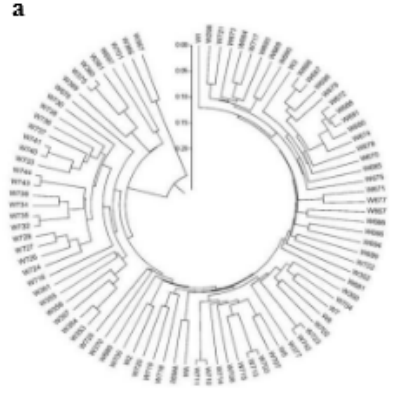

b

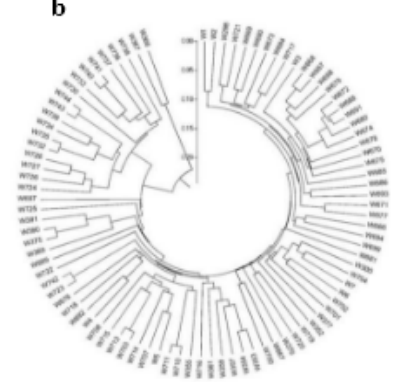

c

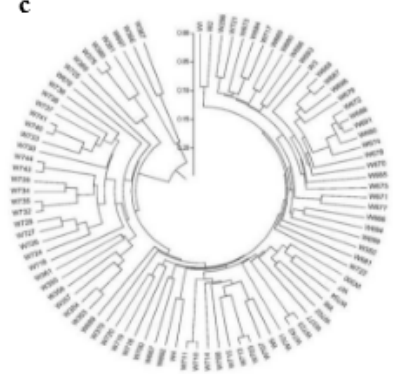

Figure 6

UPGMA Clustering using different SNPs markers. a. by SNPs-Nlalll; b. by SNPs-Msel; c. by merged datas of SNPs-Nlalll plus SNPs-Msel
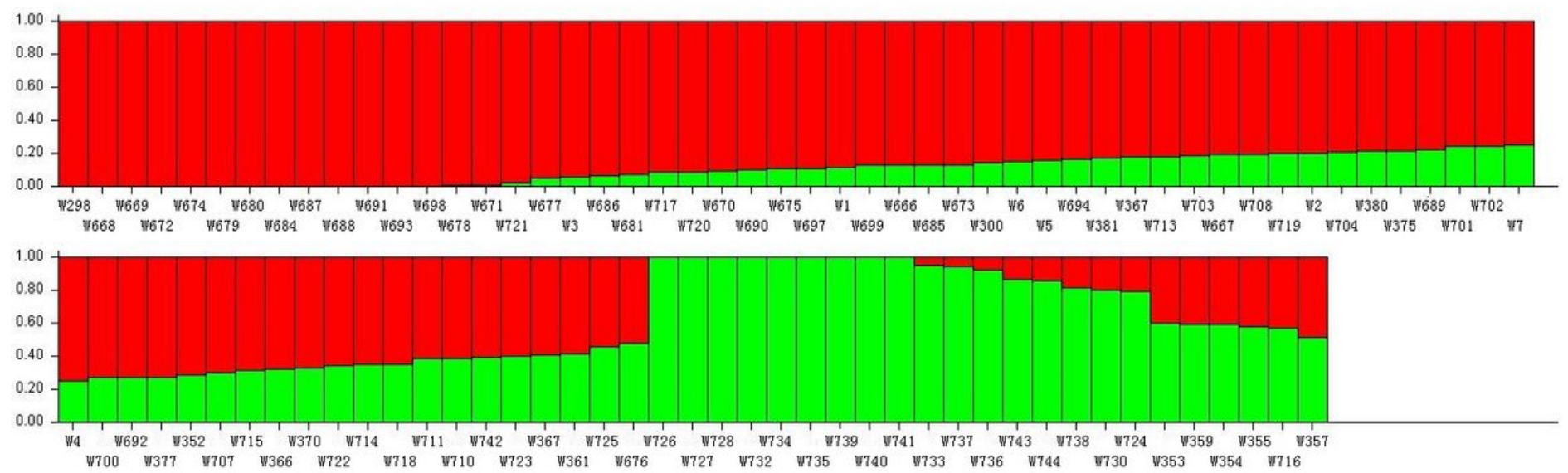

\section{Figure 7}

Bayesian clustering based on 72,824 SNPs for 93 samples; Red: group I; Green: group II. Each vertical line on the X-axis correspond to a sample. The proportion of each color represents probability rate with which a given genotype belongs to each group.

a

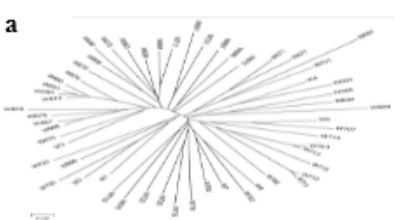

h

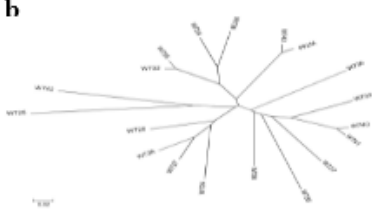

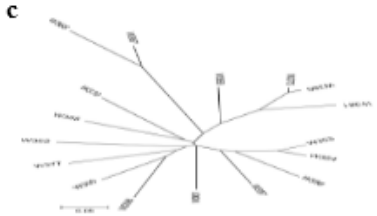

\section{Figure 8}

Clustering based on UPGMA. a. clustering of 57 restoring lines. b. clustering of 19 maintainer lines. c. clustering of 17 special rice lines. 

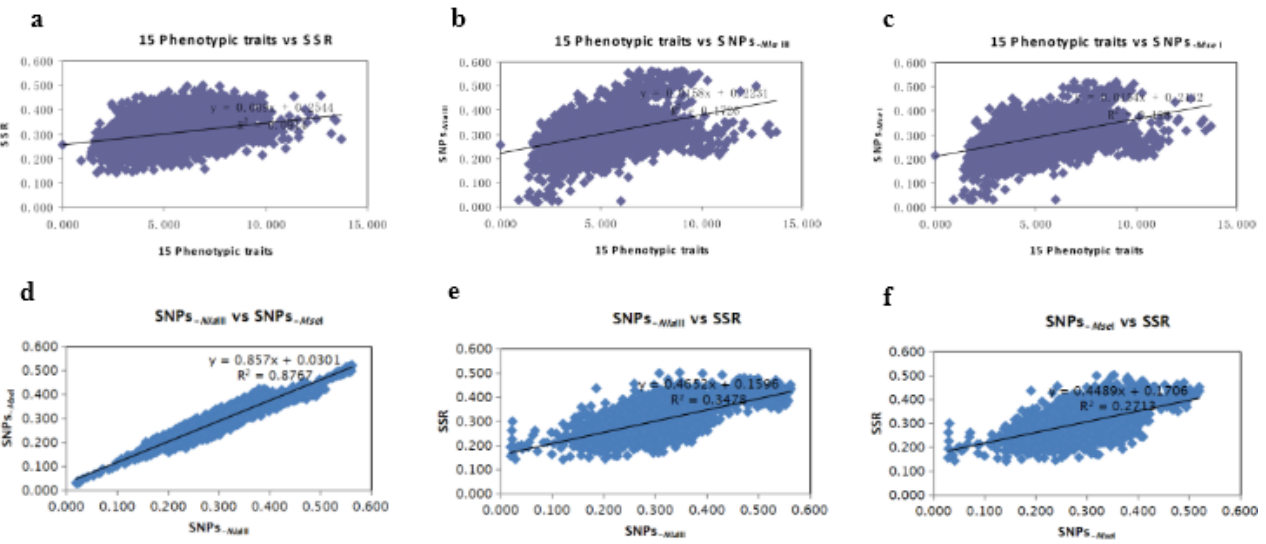

Figure 9

Correlation between the genetic distance matrices generated using different genetic markers 